\title{
Change in eyelid parameters after orbital decompression in thyroid- associated orbitopathy
}

\author{
Hong Hyun Park ${ }^{1} \cdot$ Yeoun Sook Chun ${ }^{1} \cdot$ Nam Ju Moon ${ }^{1}$ Jee Taek Kim $\mathbb{D}^{1} \cdot$ Sang Joon Park ${ }^{2,3} \cdot$ Jeong Kyu Lee $\mathbb{D}^{1}$
}

Received: 14 October 2016 / Revised: 7 November 2017 / Accepted: 15 November 2017 / Published online: 2 February 2018

(c) The Author(s) 2018. This article is published with open access

\begin{abstract}
Purpose To investigate quantitative changes in eyelid parameters after orbital decompression surgery in thyroid-associated orbitopathy using the PC-based program, Eyelid Analysis Software (EAS).

Methods This study included 202 eyes of 109 thyroid-associated orbitopathy patients. Digital photographs of the patients in primary gaze were taken just before and after orbital decompression surgery, and exophthalmos degree was measured by Hertel exophthalmometry (Hertel Exophthalmometer SKU:52400; Oculus, Arlington, VA, USA). The custom-made PCbased software EAS (Biomedical Research Institute, Seoul, Korea) was used to analyze eyelid parameters. The 11 parameters included pupil to inferior eyebrow distance (PBD), margin reflex distance 1 (MRD1), margin reflex distance 2 (MRD2), palpebral fissure (PF), total lid length, upper and lower lid length, area, medial area, center area, and lateral area. Results Univariate linear regression analysis showed a significant positive association between amount of exophthalmos reduction and the following parameters: area $(p=0.007)$; MRD2 $(p=0.043)$; upper lid length $(p=0.045)$; lower lid length $(p=0.006)$; medial area $(p=0.045)$; and lateral area $(p=0.005)$. Multivariate linear regression analysis showed only two parameters, lower lid length $(p=0.022)$ and lateral area $(p=0.019)$ were associated with exophthalmos reduction.

Conclusions A reduction in the inferior lateral part of the eyelid (lateral area + lower lid length) occurred after orbital decompression surgery in patients with thyroid-associated orbitopathy.
\end{abstract}

\section{Introduction}

Thyroid-associated orbitopathy (TAO) is an inflammatory autoimmune disorder characterized by lymphocyte infiltration of the orbit. It causes fibrosis and expansion of extraocular muscles and orbital fatty tissue [1]. These conditions often result in exophthalmos, extraocular muscle restriction, and eyelid retraction [2]. These complications

Electronic supplementary material The online version of this article (https://doi.org/10.1038/s41433-018-0022-6) contains supplementary material, which is available to authorized users.

Jeong Kyu Lee

1k1246@gmail.net

1 Department of Ophthalmology, Chung-Ang University College of Medicine, Chung-Ang University Hospital, Seoul, Korea

2 Department of Radiology, Seoul National University College of Medicine, Seoul, Korea

3 Biomedical Research Institute, Seoul National University Hospital, Seoul, Korea can be problematic for daily life or have a serious effect on visual acuity and can necessitate medical or surgical treatment. General surgical treatment options include orbital decompression with or without removal of fat, eyelid surgery, and strabismus surgery $[3,4]$.

Empirically, we know that orbital decompression surgery in TAO not only reduces exophthalmos but also changes eyelid contour and position to some extent. Previous studies have reported that orbital decompression surgery can reduce lower eyelid retraction [5, 6]. However, those studies have manually measured the eyelid contour and are limited to linear measurement, such as margin reflex distance 1 (MRD1; the vertical distance between the light reflex of the subject's cornea to the upper eyelid margin center), margin reflex distance 2 (MRD2; the vertical distance between the light reflex to the lower eyelid margin center), and palpebral fissure (PF; the vertical height between the eyelids). With the same value for MRD1, MRD2, and PF, even the contour and length of the eyelid can appear very different, for this reason these parameters are insufficient for quantitatively expressing eyelid changes after orbital decompression surgery. Therefore, we used a PC-based program Eyelid 
Fig. 1 Schematic drawing of eyelid parameters; upper left: MRD1 and MRD2. Upper right: upper and lower lid length. Lower left: area and PBD. Lower right: medial area, center area, and lateral area. MRD1 = margin reflex distance from the corneal light reflex to the upper eyelid margin; MRD2 $=$ margin reflex distance from the corneal light reflex to the lower eyelid margin; palpebral fissure $=$ MRD1 + MRD2; total lid length = upper lid length + lower lid length; $\mathrm{PBD}=$ pupil to inferior eyebrow distance; area $=$ medial area + center area + lateral area
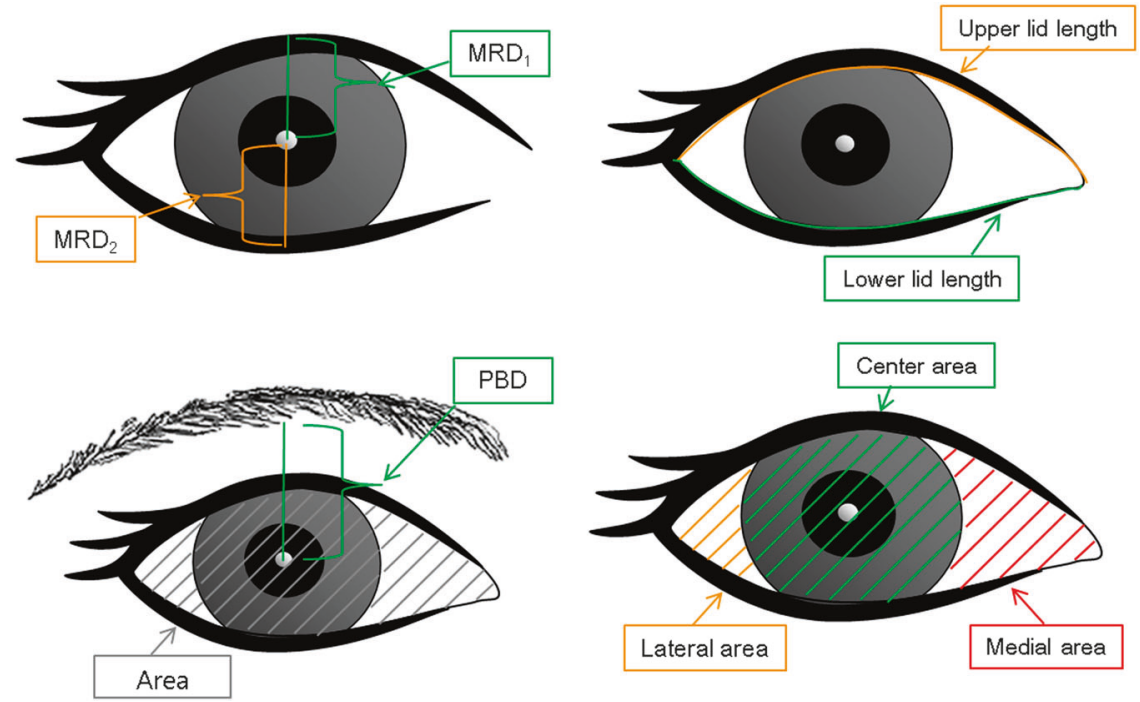

Analysis Software (EAS), which can quantify eyelid parameters and variously express the eyelid contour. We used EAS to perform a more detailed analysis of eyelid contour change after orbital decompression surgery.

\section{Materials and methods}

\section{Patients and measurements}

This retrospective study adhered to the tenets of the Declaration of Helsinki and was approved by the ChungAng University Hospital Institutional Review Board. All orbital decompression surgeries were performed by the authors from January 2011 to December 2014, and were retrospectively reviewed. Just before the surgeries, and within 3-6 months after the surgeries, digital photos were taken for all. All photos were taken using a digital camera (Nikon D90 digital camera; Nikon, Tokyo, Japan) with a standardized technique. Using this technique, the patients' heads were placed in the modified slit lamp to position their heads consistently in the same plane in relation to the camera and to generate standardized images of the eyelids. Patients were instructed to relax and to fixate on the center of the camera lens with their eyes in a primary gaze position. Also, the degree of exophthalmos was measured by Hertel exophthalmometry (Hertel Exophthalmometer SKU:52400; Oculus, Arlington, VA, USA).

Based on the degree of exophthalmos, the appropriate surgical method was selected, including balanced decompression (medial and deep lateral wall decompression) and three-wall decompression (medial, inferior, and deep lateral wall), with or without fat removal. The medial wall was approached through a transcaruncular incision, the inferior wall was approached through a transconjunctival incision, and the lateral wall was approached through an eyelid crease incision. Custom-made PC-based software EAS (Biomedical Research Institute, Seoul, Korea) was used to analyze eyelid parameters. After the digital images were loaded into the software, the brow to pupil distance, the vertical distance between the inferior eyebrow and the pupil center, was measured. Then, eyelid segmentation was performed using an interactive-polygonal drawing interface. Next, MRD1, MRD2, PF, total lid length, upper and lower lid lengths, area, medial area, center area, and lateral area were automatically computed (Fig. 1).

Criteria for inclusion were as follows: orbital decompression surgery due to TAO, clinically stable state for 6 months or more before surgery, and postoperative followup for at least 3 months. Criteria for exclusion were the following: previous ocular surgery especially eyelid surgery or orbital decompression surgery for cosmetic purposes without TAO. If the patients underwent eyelid or strabismus surgery after orbital decompression surgery, the day just before receiving eyelid or strabismus surgery was set as the last follow-up.

\section{Statistical analysis}

Data were expressed as mean $\pm \mathrm{SD}$ unless otherwise indicated.

Paired $t$-tests were used to compare changes in eyelid parameters before and after orbital decompression surgery. Parameter change amount according to surgical procedures was compared using one-way analysis of variance (ANOVA). The relationships between the amount of exophthalmos reduction and changes in eyelid parameters after orbital decompression surgery were assessed by univariate and multivariate regression analyses. Through the regression analyses, independent parameters for the association with 
Table 1 Characteristics of patients who underwent orbital decompression ( $N=109$ patients, 202 eyes)

\begin{tabular}{lc}
\hline Characteristics & Value \\
\hline Age $(y)$, mean \pm SD & $32.6 \pm 10.5$ \\
Sex, $N(\%)$ & \\
$\quad$ Male & $31(28.4)$ \\
Female & $78(71.6)$ \\
Surgical site, $N(\%)$ & \\
Bilateral & $93(85.3)$ \\
Unilateral & $16(14.7)$ \\
Smoking, $N(\%)$ & $55(27.2)$ \\
Laterality, $N(\%)$ & \\
Right eye & $100(49.5)$ \\
Left eye & $102(50.5)$ \\
Ocular examination, $N(\%)$ & \\
Upper lid retraction & $94(46.5)$ \\
Lid lag & $62(30.7)$ \\
Lagophthalmos & $47(23.3)$ \\
Scleral show & $105(52)$ \\
Exophthalmometry $(\mathrm{mm}, \mathrm{Hertel}) \pm \mathrm{SD}$ & $p<0.001^{\mathrm{a}}$ \\
Preoperation & $19.9 \pm 2.1$ \\
Post operation & $15.6 \pm 1.9$ \\
\hline
\end{tabular}

${ }^{\mathrm{a}} p$-values; significance set at $<0.05$

exophthalmos reduction were determined, and the equation between the parameters was calculated. Statistical analyses were performed using SPSS software version 19.0 (PASW, ver. 19.0; SPSS Inc., Chicago, IL, USA). The alpha level (type I error) was set at 0.05 .

\section{Results}

This study enrolled a total of 137 TAO patients who underwent orbital decompression surgery. Among them, 28 patients were excluded from the study because of the following reasons: (1) the patients did not or could not come to the clinic after orbital decompression surgery; and (2) poor image quality. Following exclusion, 109 patients were available, and 202 eyes were included in the analysis. The baseline characteristics are presented in Table 1 . Table 2 shows the changes in eyelid parameters after orbital decompression surgery. Ten parameters except $\operatorname{PBD}(p=$ 0.093 ) measured by the software showed statistically significant reductions after surgery. Also, we evaluated whether the parameter values changed depending on the surgical technique using the one-way ANOVA method. All parameters were not yield statistically significant (Supplementary Table 3).

After orbital decompression surgery, exophthalmos is necessarily reduced. Our study determined the "degree of
Table 2 Parameter changes after orbital decompression surgery $(N=$ 202 eyes)

\begin{tabular}{|c|c|c|c|}
\hline Parameter & $\begin{array}{l}\text { Pre-OP (mean } \pm \\
\text { SD) }\end{array}$ & $\begin{array}{l}\text { Post-OP (mean } \\
\pm \mathrm{SD})\end{array}$ & $p$-value ${ }^{\mathrm{a}}$ \\
\hline PBD (mm) & $16.7 \pm 1.6$ & $16.1 \pm 1.9$ & 0.093 \\
\hline Area $\left(\mathrm{mm}^{2}\right)$ & $190.5 \pm 41.7$ & $165.5 \pm 33.0$ & $<0.001$ \\
\hline $\begin{array}{l}\text { Total lid length } \\
(\mathrm{mm})\end{array}$ & $63.0 \pm 4.9$ & $59.2 \pm 4.2$ & $<0.001$ \\
\hline MRD1 (mm) & $3.7 \pm 1.4$ & $3.5 \pm 1.1$ & 0.001 \\
\hline MRD2 (mm) & $6.3 \pm 1.0$ & $5.7 \pm 1.0$ & $<0.001$ \\
\hline $\begin{array}{l}\text { Palpebral fissure } \\
(\mathrm{mm})\end{array}$ & $10.1 \pm 1.8$ & $9.2 \pm 1.4$ & $<0.001$ \\
\hline $\begin{array}{l}\text { Upper lid length } \\
(\mathrm{mm})\end{array}$ & $33.6 \pm 2.6$ & $31.4 \pm 2.4$ & $<0.001$ \\
\hline $\begin{array}{l}\text { Lower lid length } \\
(\mathrm{mm})\end{array}$ & $29.7 \pm 3.1$ & $27.8 \pm 2.0$ & $<0.001$ \\
\hline Medial area $\left(\mathrm{mm}^{2}\right)$ & $50.1 \pm 17.5$ & $39.9 \pm 14.6$ & $<0.001$ \\
\hline Center area $\left(\mathrm{mm}^{2}\right)$ & $85.5 \pm 11.8$ & $81.7 \pm 10.3$ & $<0.001$ \\
\hline Lateral area $\left(\mathrm{mm}^{2}\right)$ & $54.5 \pm 19.8$ & $43.6 \pm 14.2$ & $<0.001$ \\
\hline
\end{tabular}

MRD1 marginal reflex distance 1; MRD2 marginal reflex distance 2; $P B D$ pupil to inferior eyebrow distance

${ }^{\text {a }}$ Paired $t$-test, significance set at $<0.05$

exophthalmos reduction" as a value that represented the orbital decompression surgery effect. Table 3 summarizes the results of univariate linear regression analysis of the associations between amount of exophthalmos reduction and changes in eyelid contour parameters. The analysis indicated a significant positive association between amount of exophthalmos reduction and the following parameters: area $(p=0.007)$; MRD2 $(p=0.043)$; upper lid length $(p=$ $0.045)$; lower lid length $(p=0.006)$; medial area $(p=$ $0.045)$; and lateral area $(p=0.005)$. Interestingly, there was no association between exophthalmos reduction and MRD1 change $(p=0.974)$, even after precluding 94 eyes, which had upper eyelid retraction before orbital decompression; also this was not statistically significant (data are not shown). The factors found to be significant in univariate analysis were entered in the multivariate analysis. The results of this analysis are shown in Table 3. This result showed that the main eyelid parameters associated with exophthalmos reduction were lower lid length $(p=0.022)$ and lateral area $(p=0.019)$. Thus, other parameters, including MRD2, appeared to be indirectly affected by exophthalmos reduction. The equation that accounted for exophthalmos changes before and after orbital decompression surgery was as follows: exophthalmos change $=3.967$ $+0.019 \times$ lateral area change $+0.099 \times$ lower lid length change. Overall, these results suggest that after orbital decompression, the outer portion of the lower eyelid was elevated in proportion to the degree of exophthalmos reduction. 
Table 3 Univariate and multivariate analyses of the association between exophthalmos and eyelid contour parameters

\begin{tabular}{|c|c|c|c|c|c|c|}
\hline \multirow[t]{2}{*}{ Parameter } & \multicolumn{3}{|c|}{ Univariate analysis } & \multicolumn{3}{|c|}{ Multivariate analysis } \\
\hline & $B$ coefficient & $\beta$ coefficient & $p$-value & $B$ coefficient & $\beta$ coefficient & $p$-value \\
\hline Area $\left(\mathrm{mm}^{2}\right)$ & 0.011 & 0.189 & 0.007 & - & 0.039 & 0.753 \\
\hline Total lid length (mm) & 0.049 & 0.120 & 0.088 & - & - & - \\
\hline MRD1 (mm) & -0.003 & -0.002 & 0.974 & - & - & - \\
\hline MRD2 (mm) & 0.245 & 0.142 & 0.043 & - & 0.037 & 0.634 \\
\hline Palpebral fissure (mm) & 0.13 & 0.105 & 0.137 & - & - & - \\
\hline Upper lid length (mm) & 0.114 & 0.141 & 0.045 & - & 0.017 & 0.834 \\
\hline Lower lid length (mm) & 0.118 & 0.193 & 0.006 & 0.099 & 0.162 & 0.022 \\
\hline Medial area $\left(\mathrm{mm}^{2}\right)$ & 0.017 & 0.141 & 0.045 & - & 0.039 & 0.620 \\
\hline Center area $\left(\mathrm{mm}^{2}\right)$ & 0.01 & 0.049 & 0.493 & - & - & - \\
\hline Lateral area $\left(\mathrm{mm}^{2}\right)$ & 0.022 & 0.196 & 0.005 & 0.019 & 0.165 & 0.019 \\
\hline
\end{tabular}

$B$ beta unstandardized coefficient; $\beta$ standardized coefficient; MRD1 marginal reflex distance 1; MRD2 marginal reflex distance 2

\section{Discussion}

Inflammatory reaction in patients with TAO produces hydrophilic hyaluronan that accumulates between the extraocular muscle fibers and the orbital adipose tissues. This leads to increased orbital volume and exophthalmos [7]. Among TAO patients, eyelid shape becomes the primary source of complaints citing drastic shape changes. Simply, due to volume increases in orbital contents and exophthalmos, eyelid contours may change. However, the eyelid contour in TAO patients is not determined only by such a mechanical factor. Chronic inflammation, particularly in upper and lower eyelid retraction, that results in fibrosis of the orbital fascia and suspensory system seems to be the main mechanism of eyelid contour change in TAO $[8,9]$. Additionally, lateral flare, which is a characteristic sign in TAO, occurs because the lateral horn of the levator palpebre superioris muscle is more forceful than the medial horn of the levator palpebre superiosis or lateral extension of Muller's muscle [10]. Thus, the eyelid contour change in TAO is caused by both mechanical and pathological effects. This study was conducted to find out how the eyelid contour varies after orbital decompression surgery.

There are a limited number of studies that have assessed orbital decompression and the effect on eyelid contour. Kikkawa et al. [6] reported that an average of $5.7 \mathrm{~mm}$ exophthalmos reduction leads to a $1.3 \mathrm{~mm}$ upper eyelid retraction reduction and a $1.6 \mathrm{~mm}$ lower eyelid retraction reduction. It is significant that MRD1 was measured after orbital decompression according to the degree of exophthalmos. However, the conventional MRD1 value was manually measured and has low reproducibility and there was no statistical correlation analysis between the degree of exophthalmos and retraction reduction. Cho et al. [5] reported a study of 165 eyes that showed a positive effect of orbital decompression on lower eyelid retraction but, not on upper eyelid retraction. Statistically, a $5 \mathrm{~mm}$ exophthalmos reduction would result in a $0.6 \mathrm{~mm}$ reduction in lower eyelid retraction. However, because the value of the eyelid retraction can be affected by patient posture, the results have limited application because the analysis did not use standardizing photography with a fixed head position. Chang et al. [11] reported that orbital decompression surgery leads to upper eyelid contour normalization. According to their study, when a $3.5 \mathrm{~mm}$ exophthalmos reduction was made, $56.12 \mathrm{~mm}^{2}$ of exposed eyelid area was reduced and the upper eyelid peak point moved to the medial side. That study utilized NIH ImageJ software (National Institutes of Health, Springfield, USA) and digital photo images. However, the study only measured two parameters, the exposed area and upper eyelid peak point, which are insufficient to represent the entire eyelid. Additionally, the study only included 38 eyes, which was a small sample size for evaluating the relationship between exophthalmos and "some" parts of the eyelid contour.

This study objectively evaluated the entire eyelid contour using PC-based software. We measured various parameters in more than 200 eyes. Univariate and multivariate regression analyses were used to determine the statistical significance of the parameters. Our multivariate regression analysis indicated that reduction in lower lid length and lateral area after orbital decompression correlated with amount of exophthalmos reduction. This suggests that the outer portion of the lower eyelid rose above its previous level after orbital decompression (Fig. 2). Thus, our results differ from previous studies [5, 6, 11]. According to those results, an improvement in eyelid retraction was noted in either the upper eyelid, lower eyelid, or both after orbital decompression surgery. Our univariate analysis showed that lower eyelid retraction was reduced after orbital decompression surgery. However, our multivariate analysis showed that there was no direct relationship between orbital 
Fig. 2 Schematic drawing and photographs of eyelid contour change before and after orbital decompression surgery; the upper is before surgery, the lower is after surgery, and a decreased in the inferior lateral surface area is indicated
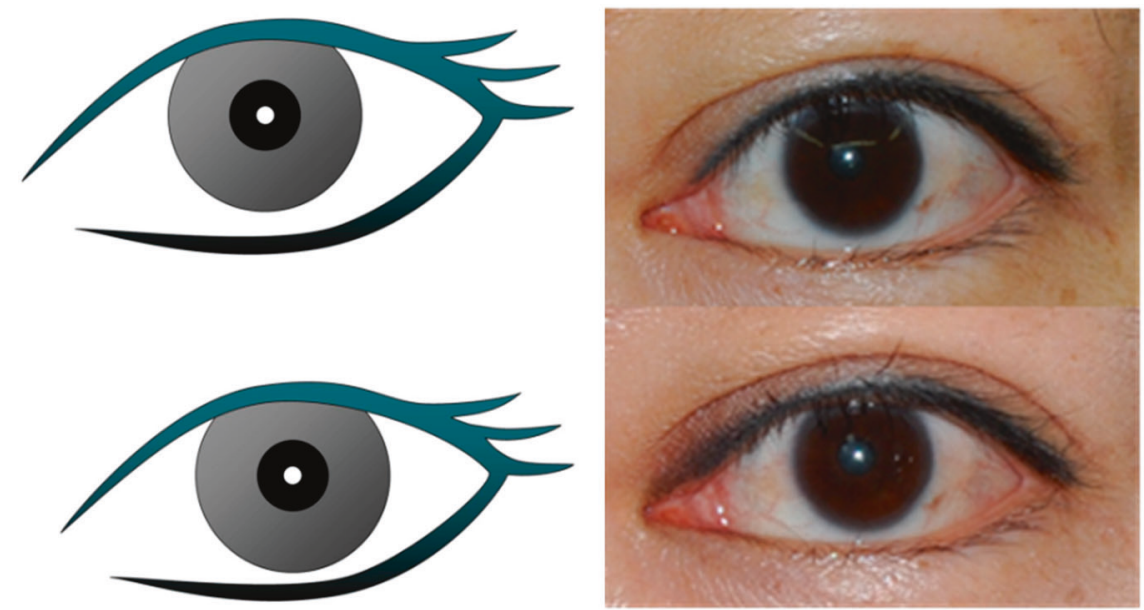

decompression surgery and eyelid retraction. There are several possible reasons for this result; first, the studies by Kikkawa et al. [6] and Cho et al. [5] used manual parameters such as MRD1 and MRD2, whereas our study used computerized parameters, which offer not only greater accuracy and reproducibility but also express eyelid contour more specifically. Second, because orbital decompression surgery was performed in a stabilized chronic phase when active inflammation had already resolved, fibrosis probably had a greater influence on upper and lower eyelid retraction than expected. Therefore, parameters expressed in MRD1 and MRD2 did not demonstrate statistically significant relationships to orbital decompression.

This study has some limitations. Due to its retrospective nature, a selection bias might have been introduced through the inclusion of certain eyes. Since we studied only Koreans who had undergone surgery at a tertiary university hospital, the results may not apply to other ethnicities. Although extraocular muscles and orbital fat can also affect the position of the eyelid, photographs alone used in this study were insufficient to account for all variables in aggregate. Further, van den Bosch et al. [12] reported eyeball position descended after orbital decompression surgery. Certainly, the exact position of the eyeball can only be ascertained through three-dimensional analysis. In this study, we did not measure eye position three-dimensionally. However, we did not find any statistically significant changes in vertical eye position before and after orbital decompression as an additional measurement of PBD. Thus, it seems that in general, eyelid contours change after orbital decompression as opposed to the eyeball position itself changing. In addition, the surgical techniques performed in this study varied from balanced decompression to three-wall decompression but conventional lateral wall decompression was not included. Since conventional decompression removes the orbital rim, unlike other surgical techniques, it can affect the periorbital structure. Therefore, further studies are warranted to evaluate the correlation between conventional surgical technique and eyelid contour changes.

In conclusion, we demonstrated that the outer portion of the lower eyelid is elevated after orbital decompression surgery in patients with TAO. Therefore, clinicians can now inform clinically stable patients that orbital decompression does not necessarily improve eyelid retraction and thus, further eyelid surgeries could be required. Thus, a detailed preoperative consultation is essential for patients scheduled for orbital decompression surgery.

\section{Summary}

\section{What was known before}

- Objective assessment of eyelid parameter is essential for identification of therapeutic effect, and communication in multicenter research intervention,several recent studies were used objective assessment tools for expression of eyelid position, such as NIH ImageJ software (National Institutes of Health, Springfield, USA), however, their analysis needed multiple steps and clicks, and their results were restricted to simple parameters.

\section{What this study adds}

- Using Eyelid Analysis software program, we attempt to quantitative analysis of the effect of orbital decompression surgery for changing in eyelid contour.

Acknowledgements This research was supported by Basic Science Research Program through the National Research foundation of Korea (NRF) funded by the Ministry of Education (NRF2015R1D1A1A01060016). The sponsor had no role in the design or conduct of this study. 


\section{Compliance with ethical standards}

Conflict of interest The authors declare that they have no competing interests.

Open Access This article is licensed under a Creative Commons Attribution-NonCommercial-NoDerivatives 4.0 International License, which permits any non-commercial use, sharing, distribution and reproduction in any medium or format, as long as you give appropriate credit to the original author(s) and the source, and provide a link to the Creative Commons license. You do not have permission under this license to share adapted material derived from this article or parts of it. The images or other third party material in this article are included in the article's Creative Commons license, unless indicated otherwise in a credit line to the material. If material is not included in the article's Creative Commons license and your intended use is not permitted by statutory regulation or exceeds the permitted use, you will need to obtain permission directly from the copyright holder. To view a copy of this license, visit http://creativecommons.org/licenses/by-nc-nd/4.0/.

\section{References}

1. Bahn RS. Graves' ophthalmopathy. N Engl J Med. 2010;362: 726-38.

2. Sergott RC, Glaser JS. Graves' ophthalmopathy. A clinical and immunologic review. Surv Ophthalmol. 1981;26:1-21.

3. Leone CR Jr.. The management of ophthalmic Graves' disease. Ophthalmology. 1984;91:770-9.
4. Mourits MP, Prummel MF, Wiersinga WM, Koornneef L. Clinical activity score as a guide in the management of patients with Graves' ophthalmopathy. Clin Endocrinol (Oxf). 1997;47:9-14.

5. Cho RI, Elner VM, Nelson CC, Frueh BR. The effect of orbital decompression surgery on lid retraction in thyroid eye disease. Ophthal Plast Reconstr Surg. 2011;27:436-8.

6. Kikkawa DO, Pornpanich K, Cruz RC Jr., Levi L, Granet DB. Graded orbital decompression based on severity of proptosis. Ophthalmology. 2002;109:1219-24.

7. Burch HB, Wartofsky L. Graves' ophthalmopathy: current concepts regarding pathogenesis and management. Endocr Rev. 1993;14:747-93.

8. Pacheco EM, Guyton DL, Repka MX. Changes in eyelid position accompanying vertical rectus muscle surgery and prevention of lower lid retraction with adjustable surgery. J Pediatr Ophthalmol Strabismus. 1992;29:265-72.

9. Lemke BN. Anatomic considerations in upper eyelid retraction. Ophthal Plast Reconstr Surg. 1991;7:158-66.

10. Morton AD, Elner VM, Lemke BN, White VA. Lateral extensions of the Muller muscle. Arch Ophthalmol. 1996;114:1486-8.

11. Chang EL, Bernardino CR, Rubin PA. Normalization of upper eyelid height and contour after bony decompression in thyroidrelated ophthalmopathy: a digital image analysis. Arch Ophthalmol. 2004;122:1882-5.

12. van den Bosch WA, Tjon-Fo-Sang MJ, Lemij HG. Eyeball position in Graves orbitopathy and its significance for eyelid surgery. Ophthal Plast Reconstr Surg. 1998;14:328-35. 